\title{
Desarrollo de una proteína recombinante fimbrial F17 de Escherichia coliy respuesta inmune frente a células mononucleares periféricas sanguíneas (PBMC) de alpaca
}

\section{Development of the recombinant fimbrial protein F17 of Escherichia coli and the immune response in alpaca peripheral blood mononuclear cells (PBMC)}

\author{
Juan Siuce M. ${ }^{1}$, Jorge Maximiliano G. ${ }^{1}$, Raquel Hurtado C. ${ }^{1}$, Raúl Rosadio A. ${ }^{1}$, \\ Lenin Maturrano H. ${ }^{1,2,3}$
}

\section{Resumen}

Escherichia coli está asociada a diarreas que pueden producir la muerte en crías de alpacas de temprana edad. Patotipos de E. coli utilizan las fimbrias para adherirse al hospedero en la primera etapa de la patogénesis. Por ello, el presente estudio tuvo como objetivo desarrollar y producir una proteína recombinante de la adhesina fimbrial F17 de E. coli y evaluar su inmunogenicidad en células mononucleares periféricas de sangre (PBMC) de alpaca. Se utilizó a BL21 como vector de expresión, se purificó la proteína recombinante mediante cromatografía de afinidad inmovilizada y se evaluó la producción de citoquinas de PBMC a las 48 y 72 horas posterior al desafío con F17. Los resultados indican un aumento significativo de la producción de citoquinas de Th1/Th2, predominantemente IFN- $\gamma$ e IL-4. Por lo tanto, la mezcla BL21-F17 podría ser considerada como un potencial candidato vacunal para la prevención de las diarreas en alpacas producidas por E. coli.

Palabras clave: vacuna, Escherichia coli, F17, EHEC, alpaca

${ }^{1}$ Laboratorio de Microbiología y Parasitología Veterinaria, Facultad de Medicina Veterinaria, Universidad Nacional Mayor de San Marcos, Lima, Perú

${ }^{2}$ Laboratorio de Zootecnia y Producción Agropecuaria, Facultad de Medicina Veterinaria, Universidad Nacional Mayor de San Marcos, Lima, Perú

${ }^{3}$ E-mail: amaturranoh@unmsm.edu.pe

Recibido: 17 de abril de 2020

Aceptado para publicación: 15 de febrero de 2021

Publicado: 24 de abril de 2021 
Escherichia coli is associated with diarrhoea that can cause death in young alpacas. Patotypes of $E$. coli use the fimbriae to adhere to the host in the first stage of pathogenesis. Therefore, the present study aimed to develop and produce a recombinant protein of $E$. coli fimbrial adhesin F17 and to evaluate its immunogenicity in alpaca peripheral blood mononuclear cells (PBMC). BL21 was used as an expression vector, the recombinant protein was purified by immobilized affinity chromatography and the production of PBMC cytokines was evaluated 48 and 72 hours after the challenge with F17. The results indicate a significant increase in the production of Th1/Th2 cytokines, predominantly IFN- $\gamma$ and IL-4. Therefore, the BL21-F17 mixture could be considered as a potential vaccine candidate for the prevention of diarrhoea in alpacas produced by E. coli.

Key words: vaccine, Escherichia coli, F17, EHEC, alpaca

\section{INTRODUCCIÓN}

La diarrea asociada a Escherichia coli es una enfermedad de importancia en crías de alpacas y llamas, cuya presentación está frecuentemente acompañada con septicemia. Las crías afectadas presentan profusa diarrea acuosa, letargia, deshidratación e incluso distención abdominal (Whitehead y Anderson, 2006). En alpacas y llamas se han desarrollado diversos estudios para la identificación de factores de virulencia asociados a los patotipos de E. coli presentes. Así, se han descrito los patotipos EPEC, ETEC y EHEC como los más frecuentes a partir de aislados de crías y adultos, tanto en casos de animales con diarreas (enfermos) y sin diarrea (Cid et al., 2012; Luna et al., 2012; Mori et al., 2014; Rosadio et al., 2012). A la vez, se han descrito algunos serotipos fimbriales y su asociación con rotavirus en crías de alpacas con diarreas y en alpacas clínicamente sanas (Morales y Paredes, 2007).

Uno de los primeros factores de virulencia en la patogénesis de $E$. coli son las adhesinas, que le permite a la bacteria asentarse y colonizar las células blanco y, posteriormente, eliminar toxinas o proteínas efectoras que alteran el funcionamiento normal de las células de la mucosa intestinal. Entre las adhesinas más representativas son las de tipo fimbrial, principalmente las fimbrias F4, F5, F6, F17, F41, F18, que están asociadas a los patotipos Enteropatogénicos (EPEC), y con mucha más frecuencia a los enterotoxigénicos (ETEC). En porcinos, la fimbria F18 está asociada a las E. coli productoras de Shiga toxina (STEC) (Nagy y Fekete, 1999). Sin embargo, estos patrones han mostrado variaciones particulares, tanto en aves como en bovinos, teniendo bacterias con combinaciones de factores de virulencia, tanto adhesinas como fimbrias de diferentes patotipos. Así, se ha reportado que la fimbria F17 es una familia de fimbrias y que sus variedades están asociados como principal adhesina de muchos patotipos, inclusive los necrotoxigénicos (NTEC) de reciente clasificación y los enterohemorrágicos (EHEC), cuya estructura fimbrial se compone de una base que es la proteína estructural mayor «A», mientras que la proteína estructural menor que tiene función de interacción o adhesión es la «G» (Bihannic et al., 2014). Por ello, las fimbrias han servido como candidato vacunal contra $E$ coli para la inmunización de diferentes especies (Francis y Willgohs, 1991; Luna-Pineda et al., 2016; Tiels et al., 2008; Zhang et al., 2018). 
La vacunación sigue siendo una de las principales estrategias para la prevención de diarreas. Se han desarrollado diferentes tipos de vacunas contra $E$. coli para diferentes especies, con base a células muertas o bacterinas de determinado serotipo fimbrial o somático, toxoides, así como algunas vacunas recombinantes contra determinados antígenos estructurales, hallándose diferente grado de protección (Cox et al., 2014). Entre las más difundidas y con mejores resultados en bovinos y porcinos son las orientadas contra determinados serotipos fimbriales (Hur $e t$ al., 2012). Por ello, el presente estudio buscó determinar la capacidad inmunogénica de la proteína recombinante de la adhesina fimbrial F17 de E. coli, mediante la medición de citoquinas relacionadas a Th1 y Th2, paso importante en la evaluación de un candidato vacunal para la prevención de diarreas en alpacas asociadas a E. coli.

\section{MATERIALES y Métodos}

\section{Lugar del Estudio}

El desarrollo y caracterización de la proteína F17 se llevó a cabo en las instalaciones de la Facultad de Microbiología, Inmunología $\mathrm{y}$ Bioquímica de The University of Tennessee Health Science Center, Memphis, USA, mientras que la evaluación in vitro de citoquinas se realizó en el Laboratorio de Biología y Genética Molecular de la Facultad de Medicina Veterinaria (FMV) de la Universidad Nacional Mayor de San Marcos (UNMSM), Lima, Perú. Los protocolos utilizados fueron aprobados por el Comité de Ética y Bienestar Animal de la FMVUNMSM, mediante Autorización de Ética N. ${ }^{\circ}$ 2017-003.

\section{Proteína Recombinante F17 (rF17)}

Los protocolos para la elaboración y producción de la proteína recombinante $\mathrm{F} 17$ (rF17) fueron adaptados de Gomes-Solecki et al. (2006), utilizándose el péptido señal de OspA de Borrelia burgdorferi.
Se utilizó la secuencia optimizada, acompañada del péptido señal del gen de la fimbria F17 N. ${ }^{\circ}$ de Acceso NC_012944.1 (pVir_11 F17) del banco de genes (Escherichia coli Vir68); secuencia conformada por 1032 pares de bases, el cual fue insertado en el plásmido pET-28a (Novagen). El plásmido posee genes de resistencia a la kanamicina y una secuencia de residuos de histidina, para la purificación proteica.

Se utilizó a la E. coli competente BL21 (Biolab) para la transformación y expresión proteica de $\mathrm{rF} 17$ insertado el plásmido $\mathrm{pET}$ 28a para la purificación proteica, siguiendo las instrucciones del fabricante.

\section{Vector y Expresión de rF17}

BL21 conteniendo pET-28 $8^{\mathrm{a}}$, plásmido codificante de rF17 (BL21+F17), fue cultivado en el medio TBY (caldo triptona y levadura) con kanamicina a una concentración final de $50 \mathrm{mg} / \mathrm{ml}$, durante más de $12 \mathrm{~h}$ a $37 \mathrm{p}$ $\mathrm{C}$, con agitación a $200 \mathrm{rpm}$. Una vez que el cultivo alcanzó 0.7-0.9 de densidad óptica, se añadió $0.5 \mathrm{mM}$ del inductor de expresión, isopropil- $\beta$-D-1-tiogalactopiranósido (IPTG) y se incubó durante $3 \mathrm{~h}$ con las condiciones previas. Luego, se centrifugó el cultivo a 4000 rpm durante $8 \mathrm{~min}$, se descartó el sobrenadante y se añadió $5 \mathrm{ml}$ de glicerol al $20 \%$ por litro de cultivo, y se almacenó a $-80^{\circ} \mathrm{C}$. Se utilizó un método estándar para la caracterización proteica (Gomes-Solecki et al., 2006).

\section{Purificación de rF17}

Se estandarizó un protocolo para la purificación del antígeno vacunal recombinante rF17: utilizando tres soluciones: Solución de Unión: fosfato de sodio $0.1 \mathrm{M} \mathrm{pH}$ 7.5. Urea 6 $\mathrm{M}$; Solución de lavado: fosfato de sodio $0.1 \mathrm{M}$ pH 6.5. Urea $6 \mathrm{M}$; Solución de elución: fosfato de sodio $0.1 \mathrm{M} \mathrm{pH} 4.5$. Urea $6 \mathrm{M}$. Asimismo, se utilizó una columna de purificación de proteínas, y con $500 \mu \mathrm{l}$ de resina de cobalto. 
Cuadro 1. Secuencias y características de los cebadores utilizados para las evaluaciones de citoquinas

\begin{tabular}{|c|c|c|c|}
\hline Genes & Secuencias & $\begin{array}{l}\text { Productos } \\
\text { pares de } \\
\text { bases }(b p)\end{array}$ & $\begin{array}{l}\text { Temperatura } \\
\text { de } \\
\text { alineamiento } \\
\left({ }^{\circ} \mathrm{C}\right)\end{array}$ \\
\hline IFN $-\gamma$ & $\begin{array}{l}\text { 5'-ATTGTCTCCTTCTACTTCAA-3' } \\
\text { 5'-AGCGGAAGAGAAGTCAGAAT -3' }\end{array}$ & 258 & 45 \\
\hline $\mathrm{TNF} \alpha$ & $\begin{array}{l}\text { 5'-CTACTCCCAGGTCCTCCTGA-3' } \\
\text { 5'-GGTAGTTGGGCATGTTGATC-3' }\end{array}$ & 251 & 60 \\
\hline IL-2 & $\begin{array}{l}\text { 5'-AAACTCTCCAGGATGCTCAC-3' } \\
\text { 5'-GGAACTGAAGGGATCTGAAA-3' }\end{array}$ & 202 & 49 \\
\hline IL-4 & $\begin{array}{l}\text { 5'-CAAAGAACACAACTGAGAAG-3' } \\
\text { 5'-GGCTAAAGAAGATTATGAAG-3' }\end{array}$ & 203 & 46 \\
\hline IL-10 & $\begin{array}{l}\text { 5'-AAGCCTTGTCGGAGATGAC-3' } \\
\text { 5'-AGCCATGAGTGAGTTCGACA-3' }\end{array}$ & 246 & 55 \\
\hline GAPDH & $\begin{array}{l}\text { 5'-GTGAAGGTCGGAGTGAACG-3' } \\
\text { 5'-GAGATGATGACCCTCTTGGC-3' } \\
\text { 5'-CTCAAGTTGGGGGACAAAAA-3' }\end{array}$ & 356 & 60 \\
\hline
\end{tabular}

Finalmente se realizó una electroforesis para proteínas (SDS-PAGE) para la verificación de la expresión y la purificación de rF17. Además, se comprobó mediante Western Blot usando anticuerpos monoclonales contra los residuos de histidina (ThermoFisher) de las proteínas purificadas de la solución de elución.

\section{Inmunogenicidad}

Para evaluar la respuesta inmune a través de la respuesta por citoquinas se utilizó un método in vitro, en el cual se cuantifica el ARN mensajero producido por los leucocitos de sangre periférica de alpaca frente al estímulo antigénico de la proteína vacunal, el cual consta de los siguientes pasos:

\section{Obtención de leucocitos circulantes}

Para obtener células mononucleares sanguíneas periféricas (PBMC) se obtuvo sangre venosa de alpaca en un tubo de ensayo de $15 \mathrm{ml}$ conteniendo EDTA como anticoagulante. Se utilizó una solución salina (PBS 1X con pH 7.4) que se mezcló con un mismo volumen de sangre (proporción 1:1).

Se utilizó el método de centrifugación en gradiente de densidad. Para ello, 03 volúmenes del reactivo Ficoll $®$-Paque (Sigma, USA) fue mezclado con 04 volúmenes de sangre con PBS. Se realizaron 2-3 lavados de las células con PBS $1 \mathrm{X} \mathrm{pH} \mathrm{7.4.} \mathrm{Finalmen-}$ te se suspendieron en medio de cultivo RPMI 1640 con L-Glutamina (Sigma, USA). 
Para determinar la concentración celular se uniformizó cada cultivo a una concentración de células por $\mathrm{ml}(\mathrm{cel} / \mathrm{ml})$. Se tomaron $5 \mu \mathrm{l}$ del paquete celular y se mezclaron con $5 \mu l$ azul de tripán diluido al décimo con PBS (1:10). La concentración y evaluación de la viabilidad celular de determinó en una cámara de Neubauer (400X). Las células se diluyeron en RPMI 1640 con L-glutamina, adicionando $100 \mathrm{IU} / \mathrm{ml}$ de penicilina (Sigma, USA) y $10 \%$ de Suero Fetal Bovino (SFB) (Sigma, USA) inactivado por calor. Los homogenizados se colocaron en una placa de poliestireno para cultivo celular de 24 pocillos unos $1200 \mu \mathrm{l}$ de cultivo celular con una concentración final de células $1 \times 10^{6}$ células $/ \mathrm{ml}$.

\section{Evaluación de la respuesta inmune}

La respuesta de los leucocitos frente al estímulo del antígeno vacunal recombinante rF17 se evaluó con el siguiente esquema experimental:

Pocillo 1: Control celular (leucocitos circulantes).

Pocillo 2: Antígeno fimbrial-rF17 (10 ng)

Pocillo 3: BL21 (10 ng) sin antígeno fimbrial (sin plásmido)

Pocillo 4: BL21 (10 ng) + antígeno fimbrial rF17 (plásmido) (20 ng)

Las placas fueron incubadas a $37^{\circ} \mathrm{C}$. La evaluación de la expresión de ARN mensajero de las citoquinas de la respuesta inmune celular y humoral se realizó a las 48 y 72 horas, mediante un RT-PCR y la cuantificación relativa delta delta $\mathrm{Ct}(\Delta \Delta \mathrm{Ct})$. Para la evaluación de citoquinas de la respuesta inmune Th1 y Th2 de alpacas (Odbileg et al., 2005) se utilizaron los cebadores respectivos (Cuadro 1).

El RT-PCR fue realizado en un termociclador PikoReal 96 (Thermo Scientific), usando como fluoróforo Maxima SYBR Green. Se utilizó el ADNc (4 ng, aproximadamente) sintetizado a partir del ARNm obtenido durante la extracción de los cultivos celulares incubados en diferentes tiempos. Para la prueba de PCR en tiempo real se utilizaron un Maxima SYBR Green/ ROX qPCR Master Mix (2X) a concentración $1 \mathrm{X}$. En la qPCR se llevó a $50{ }^{\circ} \mathrm{C}$ durante 2 min (TDG), luego una temperatura de desnaturalización (TD) inicial de $95{ }^{\circ} \mathrm{C}$ durante $10 \mathrm{~min}$, luego 40 ciclos de desnaturalización (TD) a $95^{\circ} \mathrm{C}$ durante $35 \mathrm{~s}$, hibridación (TA) a la temperatura que corresponde a cada cebador durante $30 \mathrm{~s}$ y una extensión (TE) de $72{ }^{\circ} \mathrm{C}$ durante $35 \mathrm{~s} \mathrm{(40} \mathrm{en}$ caso de GAPDH). Finalmente, una evaluación de la temperatura de disociación (TMELT), que va desde $\left\langle\mathrm{X} »-95^{\circ} \mathrm{C}\right.$, donde «X» corresponde a la TA de cada gen evaluado.

\section{Análisis Estadístico}

Las diferencias de expresión de cada citoquina fueron evaluadas usando los valores cuantitativos de expresión de citoquinas relativos mediante los intervalos de confianza en un gráfico de barras utilizando el software Graphpad Prism 7.0 (GraphPad Software, USA).

\section{Resultados}

\section{Expresión y Purificación Proteica}

La caracterización de la proteína recombinante $\mathrm{rF} 17$ mediante la determinación de la naturaleza y ubicación de expresión del antígeno vacunal en el vector recombinante pET-28a, con base a la secuencia de nucleótidos de $35 \mathrm{Kda}$ en promedio, determinó a $\mathrm{rF} 17$ en la fase detergente, tal como se observa en la Figura 01.

Se observó un producto aproximado de $35 \mathrm{Kda}$ correspondiente a la purificación del antígeno vacunal, $\mathrm{rF17}$, como se observa en la Figura 2. La evaluación de especificidad del purificado mediante la técnica de Western Blot muestra un producto único (Figura 3). 


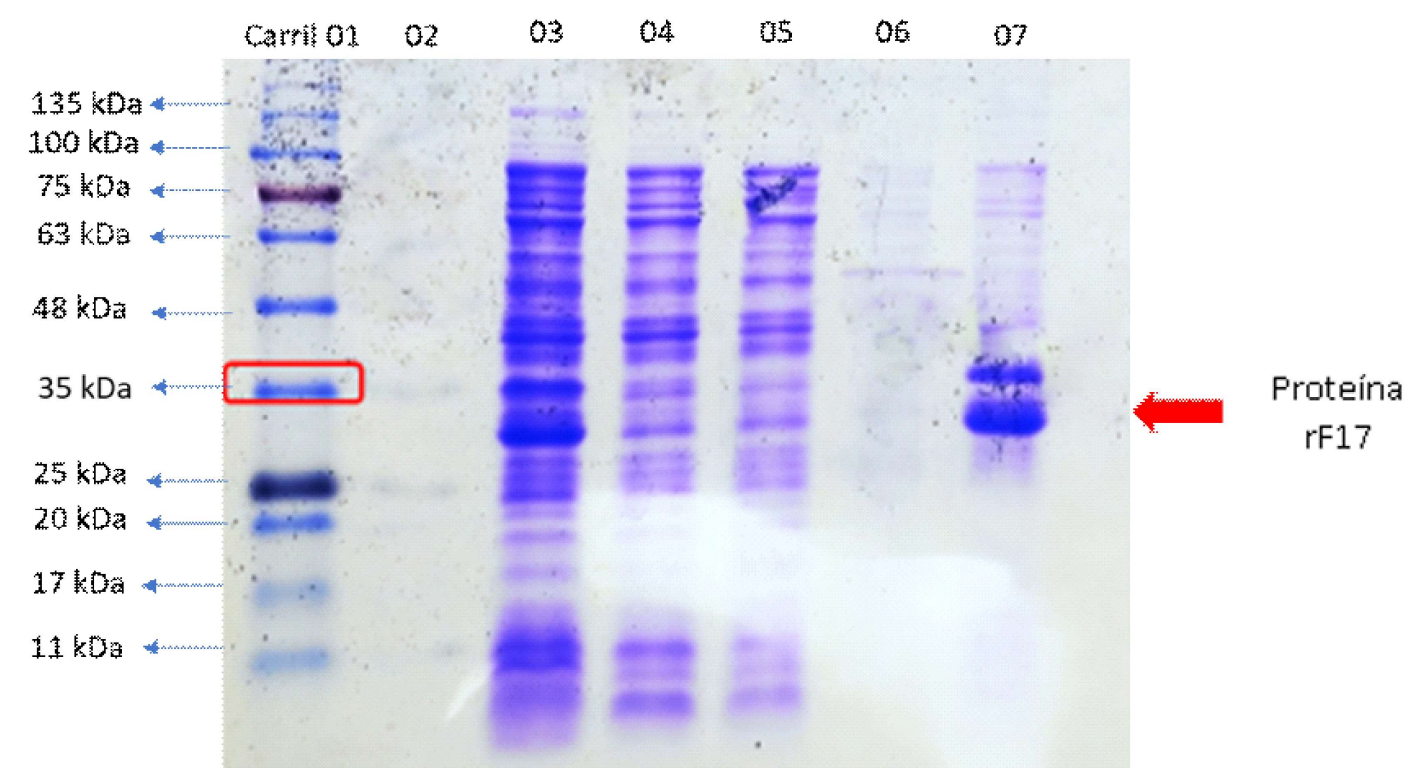

Figura 1. SDS-PAGE de la caracterización de la proteína recombinante $\mathrm{rF} 17$ usando el plásmido pET-28a. Carril 01: Marcador de peso molecular; Carril 02: blanco; Carril 03: célula completa; Carril 04: Citosol; Carril 05: Envoltura celular; Carril 06: Fracción acuosa, Carril 07: Fracción detergente. La flecha roja indica la proteína recombinante

\section{Evaluación de la Inmunogenicidad}

La cuantificación relativa de la producción de ARNm de las citoquinas Interferón gamma (IFN), Factor de Necrosis Tumoral á, IL-2, IL-4 e IL-10, estimulada por la proteína recombinante purificada $\mathrm{rF} 17$ en leucocitos de sangre periférica de alpaca (PBMC) mostraron los siguientes resultados: la mayor expresión de interferón gamma (IFN- $\gamma$ ) por leucocitos de sangre periférica de alpaca a las $48 \mathrm{~h}$ posterior a la exposición se evidenció en el grupo Vector $(3.87 \pm 0.57)$, el cual mostró niveles significativamente mayores en relación al control $(2.13 \pm 0.58)$, mientras que a las $72 \mathrm{~h}$, tanto el Vector $(5.00$ $\pm 0.90)$ como la combinación Vector $+\mathrm{rF} 17$ $(4.10 \pm 0.59)$ mostraron niveles significativamente mayores que los controles (Figura 4).
La producción de TNF- $\gamma$ a las 48 y $72 \mathrm{~h}$ mostraron niveles relativamente similares comparado con el control frente a la proteína $\mathrm{rF17}$, vector y la combinación, sin hallase diferencias significativas (Figura 5).

La expresión de la interleucina 2 (IL-2) a las 48 horas de la exposición de las proteínas $\mathrm{rF17}$, vector y la combinación mostraron niveles significativamente mayores en relación al control, siendo el vector $(6.54 \pm 1.44)$ el de mayor expresión. Sin embargo, a las 72 horas, solo el vector $(5.32 \pm 1.77)$ presentó niveles significativamente mayores (Figura 6).

En la expresión de la interleucina 4 (IL4) a las $48 \mathrm{~h}$ de la exposición, solo los grupos Vector $(5.62 \pm 1.12)$ y la combinación Vector $+\mathrm{rF} 17(4.67 \pm 0.42)$ mostraron niveles significativamente mayores con relación al 


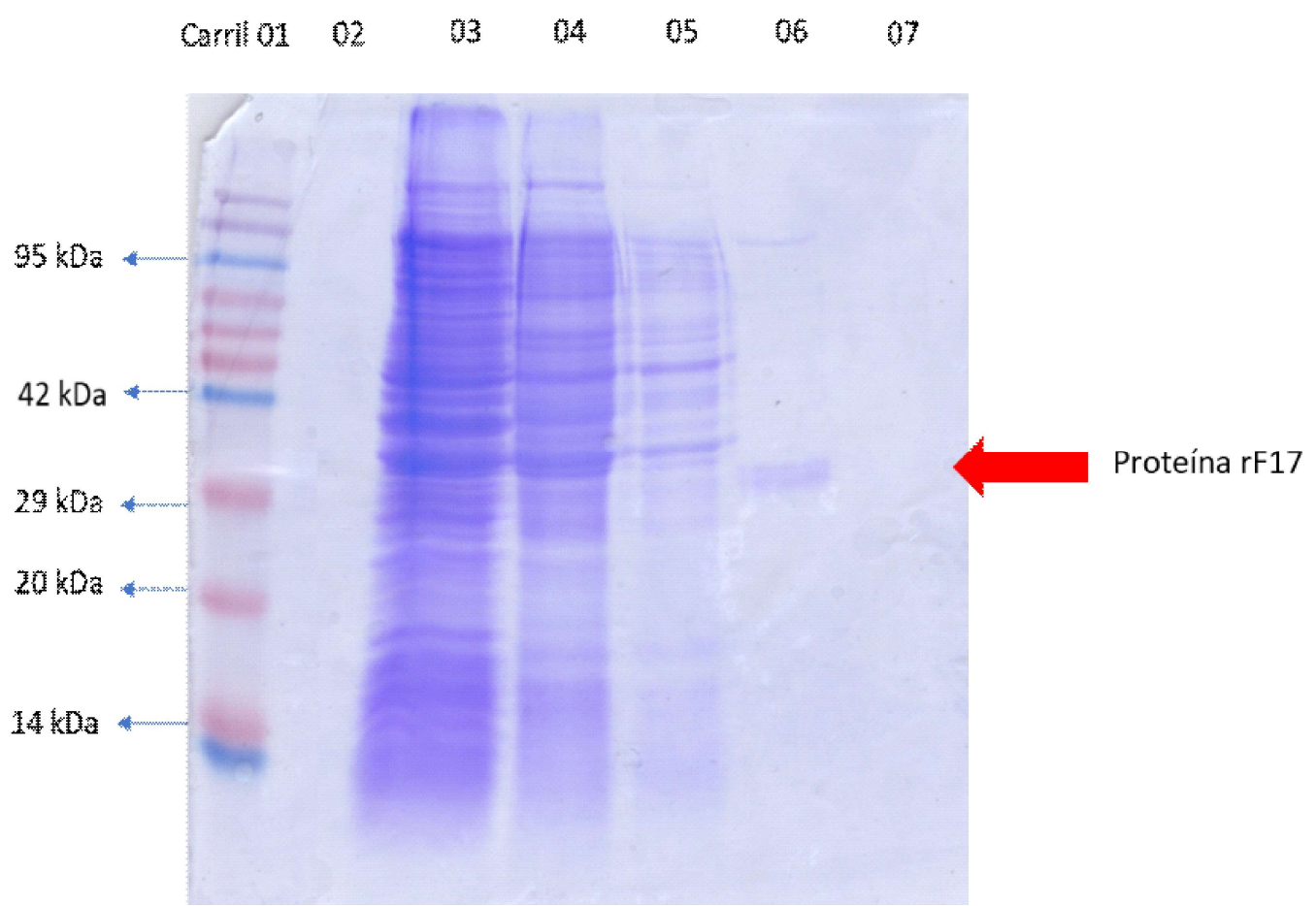

Figura 2. SDS-PAGE de la purificación de la proteína recombinante rF17. Carril 01: Marcador de peso molecular; Carril 02: blanco; Carril 03: Filtrado; Carril 04: Lavado A; Carril 05: Lavado B; Carril 06: Elusión 01, Carril 07: Elusión 02

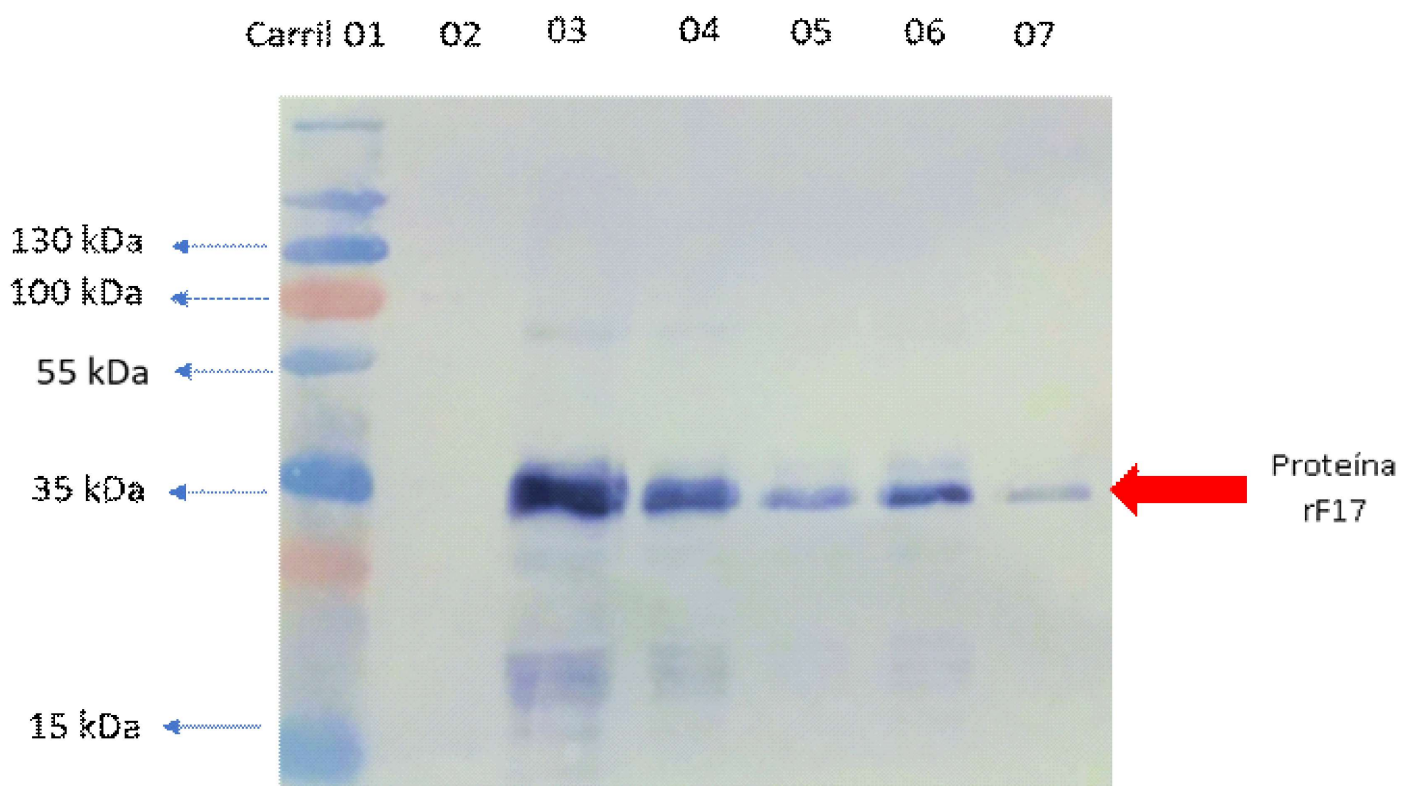

Figura 3. Western Blot usando un marcador de residuos de histidina. Carril 01: Marcador de peso molecular; Carril 02: blanco; Carril 03: Elusión 1; Carril 04: Elusión 2; Carril 05: Elusión 3; Carril 06: Elusión 4; Carril 07: Elusión 5. La flecha roja indica la proteína recombinante 
IFN - y

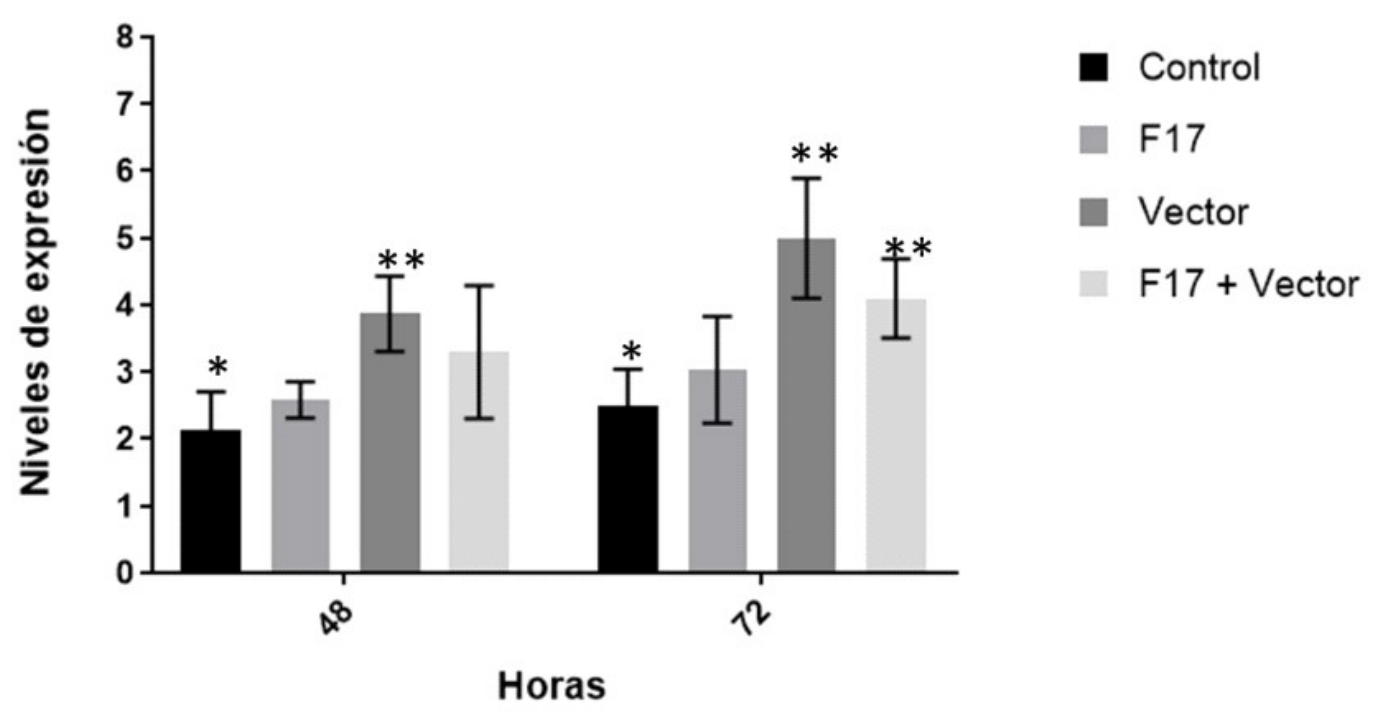

Figura 4. Niveles de expresión relativa de INF- $\gamma$ producidos por células mononucleares sanguíneas periféricas estimulados por la proteína purificada $\mathrm{rF} 17$ y componentes estructurales del vector inactivado. Las barras representan la media \pm SEM

\section{TNF- $\alpha$}

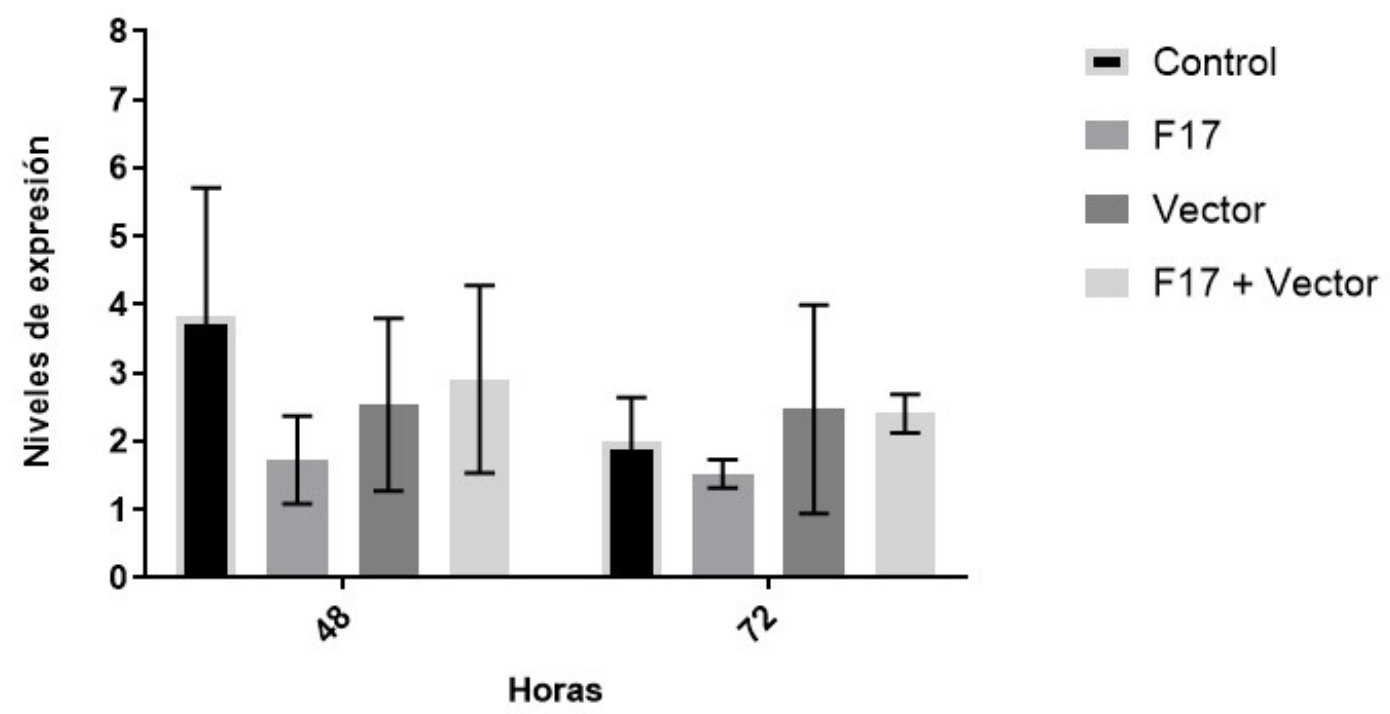

Figura 5.Niveles de expresión relativa de TNF- $\alpha$ producidos por células mononucleares sanguíneas periféricas estimulados por la proteína purificada $\mathrm{rF} 17$ y componentes estructurales del vector inactivado. Las barras representan la media \pm SEM 
IL-2

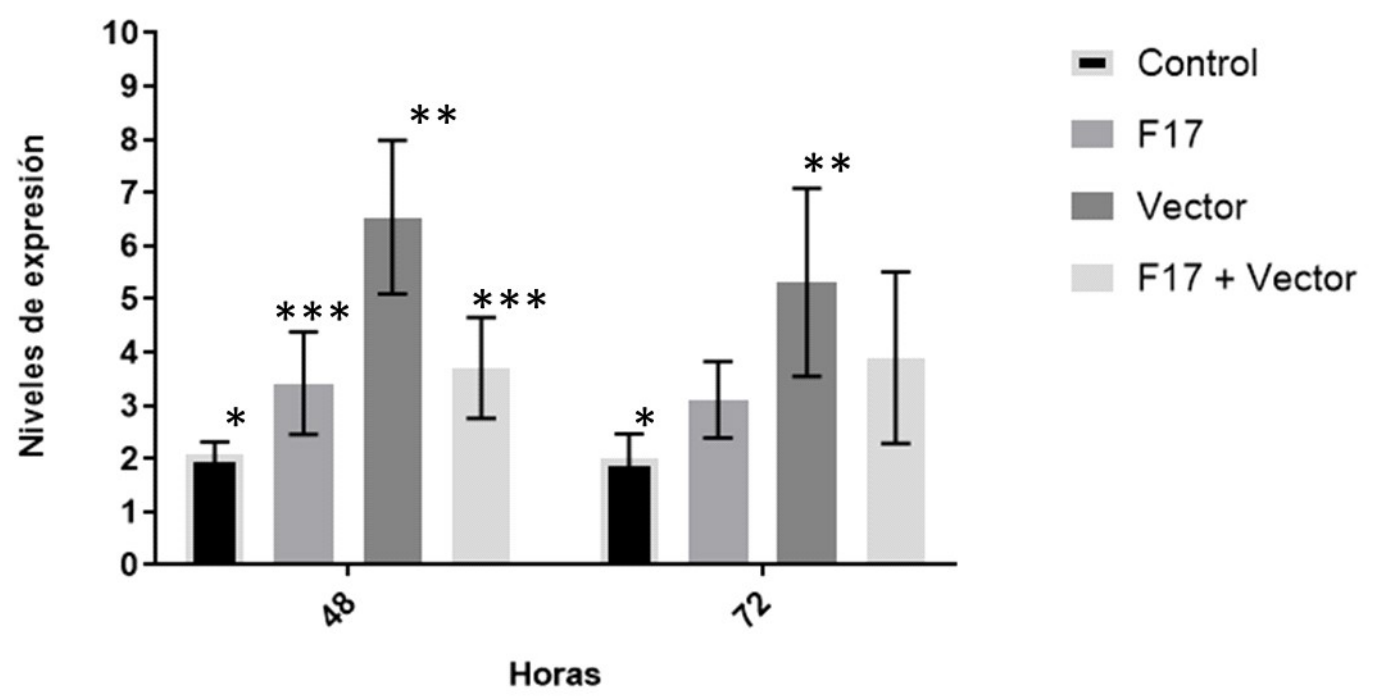

Figura 6. Niveles de expresión relativa de IL-2 producidos por células mononucleares sanguíneas periféricas estimulados por la proteína purificada F17 y componentes estructurales del vector inactivado. Las barras representan la media \pm SEM

IL-4

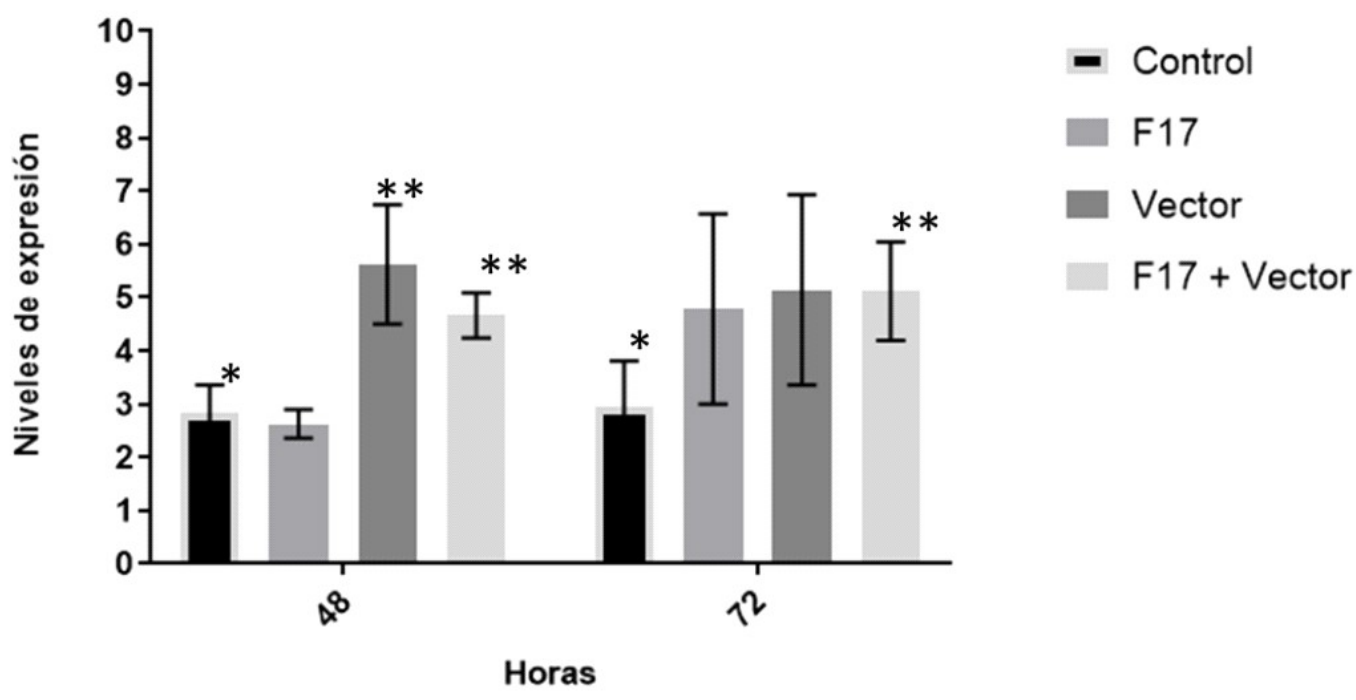

Figura 7. Niveles de expresión relativa de IL-4 producidos por células mononucleares sanguíneas periféricas estimulados por la proteína purificada $\mathrm{rF} 17$ y componentes estructurales del vector inactivado. Las barras representan la media \pm SEM 


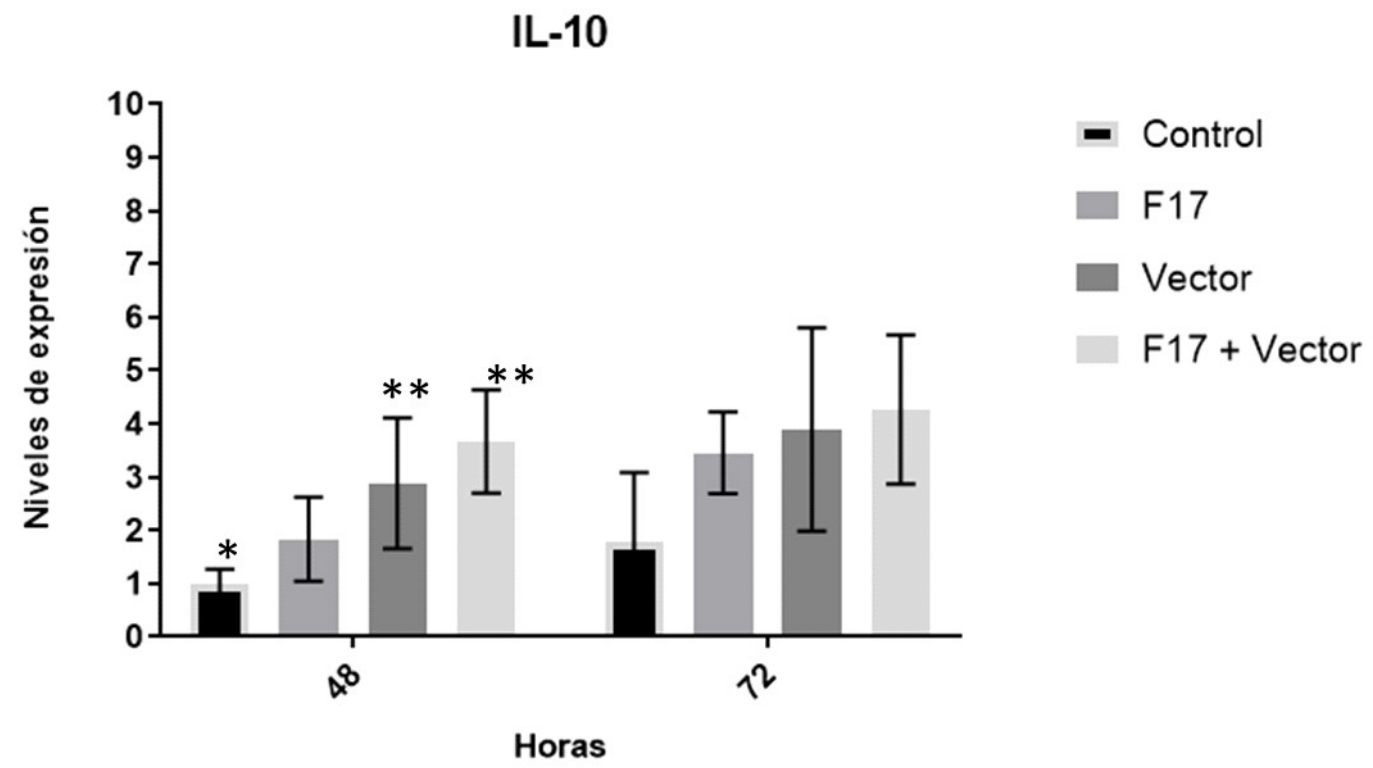

Figura 8. Niveles de expresión relativa de IL-10 producidos por células mononucleares sanguíneas periféricas estimulados por la proteína purificada $\mathrm{rF} 17 \mathrm{y}$ componentes estructurales del vector inactivado. Las barras representan la media \pm SEM

control, mientras que a las $72 \mathrm{~h}$ solo la combinación Vector $+\mathrm{rF} 17$ (5.12 \pm 0.93$)$ expresaron niveles significativamente mayores (Figura 7$)$.

La expresión de la interleucina 10 (IL10) evidenció a las $48 \mathrm{~h}$ de la exposición que solo los grupos Vector $(2.88 \pm 1.23)$ y la combinación Vector $+\mathrm{rF} 17(3.67 \pm 0.97)$ alcanzaron niveles significativamente mayores con relación al grupo control, mientras que a las $72 \mathrm{~h}$ no hubo diferencias significativas entre grupos (Figura 8).

\section{Discusión}

Se llevó a cabo la expresión de la proteína recombinante $\mathrm{F} 17$ (rF17) utilizando el vector BL21, el cual es uno de los más usados para la síntesis de proteínas recombinantes (Rosano y Ceccarelli, 2014). La producción de la proteína fimbrial $\mathrm{rF} 17$ se obtuvo utilizando el vector pET-28a, obteniéndose un producto de $35 \mathrm{Kda}$ en promedio (Figura 1). Utilizando las colas de histidina se pudo purificar la proteína (Figura 2), y para corroborar la purificación se realizó un Western Blot obteniéndose bandas únicas de $35 \mathrm{Kda}$ en todas las eluciones (Figura 3), como resultado de procedimientos estandarizados que han sido utilizados para la expresión y purificación de otras proteínas fimbriales de E. coli, como son las fimbrias de ETEC, CFA/I y CS4 (Curtis et al., 2016), F9 de EHEC del serotipo O157:H7 (Mohawk et al., 2010), e inclusive hasta la expresión de varias adhesinas fusionadas, dímeros y trímeros en E. coli uropatogénica (UPEC) (Luna-Pineda et al., 2016).

La evaluación de citoquinas y su producción frente a estímulos es una de las herramientas para la evaluación del sistema in- 
mune, siendo una de las alternativas la medición directa en suero. Sin embargo, el suero posee gran cantidad de inhibidores (proteínas dianas o receptores antagonistas y anticuerpos) (Heney y Whicher, 1995). Así, la evaluación en células mononucleares de sangre periférica (PBMC), es un método que ofrece ventajas para la evaluación de la inmunocompetencia o el estado de respuesta específica (Friberg et al., 1994; Whiteside, 1994). Este método ha sido utilizado en la evaluación de la respuesta inmune en camellos y alpacas, tanto para la evaluación de la respuesta inmune frente a inmunógenos bacterianos o proteínas simples como para la evaluación de vacunas (More et al., 2013; Tambillo et al., 2014; Watanabe et al., 2014; Sulabh et al., 2019). En bovinos, utilizando la misma metodología se ha evaluado la capacidad de resistencia y/o susceptibilidad a enfermedades de algunas razas (Sulabh et al., 2019).

En el presente estudio, la expresión de IFN-ã a las $48 \mathrm{~h}$ de la exposición el grupo Vector $(3.87 \pm 0.57)$ fue el único que presentó niveles significativamente mayores con relación al grupo control $(2.13 \pm 0.58)$; respuesta que podría estar relacionada al lipopolisacárido (LPS) que recubre la pared externa del vector (E. coli), el cual ha mostrado ser un componente que estimula la respuesta de esta citoquina (Tizard, 2013; Kleiveland, 2015). Si bien, la proteína recombinante $\mathrm{rF} 17$, por sí sola, no mostró tener la capacidad suficiente para estimular la producción de IFN- $\gamma$, al menos, hasta las 72 $\mathrm{h}$, tanto el Vector como la combinación Vector $+\mathrm{rF} 17$, mostraron niveles mayores que los controles. Estas respuestas podrían explicarse a la naturaleza de respuesta de los linfocitos, los cuales producen una mejor respuesta frente a moléculas complejas (la combinación proteína purificada con el LPS del vector). Similares respuestas de incremento de la expresión de IFN- $\gamma$ in vitro en alpacas se ha reportado usando proteínas purificadas P6 like de Pasteurella y antígenos clostridiales (More et al., 2013: Maximiliano et al., 2018).
La producción de TNF- $\alpha$ a las 48 y 72 h presentaron niveles relativamente similares al control frente a la proteína $\mathrm{rF} 17$, vector y su combinación; $\mathrm{y}$ aumentando a las $72 \mathrm{~h}$ por encima del control, aunque sin llegar a mostrar diferencias significativas. Si bien, TNF- $\alpha$ es una citoquina proinflamatoria, es una citoquina producida principalmente por células de la inmunidad innata, como son los polimorfonucleares (Tizard, 2013). Sin embargo, se debe considerar que en células de PBMC, utilizadas en este tipo de evaluaciones, se han separado a los granulocitos, siendo la respuesta obtenida por Linfocitos $\mathrm{T}$ y $\mathrm{B}$, que son células que expresan otro perfil de citoquinas (Tizard, 2013; Kleiveland, 2015). Además, su expresión puede ser inhibida por la IL-10 (Schandené et al., 1994), citoquina que fue expresada en niveles significativamente mayores en el presente estudio. Patrones similares fueron observados utilizando la proteína P6 like (Maximiliano et al., 2018) y frente a antígenos clostridiales (Tambillo et al., 2014).

La expresión de la interleucina 2 (IL-2) a las $48 \mathrm{~h}$ de la exposición de las proteínas rF17, vector y su combinación mostraron niveles significativamente mayores con relación al control, siendo el vector $(6.54 \pm 1.44)$ el de mayor expresión por encima de $\mathrm{rF} 17$ $(3.42 \pm 0.96)$ y la combinación Vector $+\mathrm{rF} 17$ (3.71 \pm 0.94$)$. Sin embargo, a las $72 \mathrm{~h}$, solo el vector $(5.32 \pm 1.77)$ presentó niveles significativamente mayores. Esta respuesta podría ser explicada debido a que IL-2 es el principal activador de Linfocitos $\mathrm{T}$, los cuales expresan el receptor de IL-2. E1 PBMC está compuesto hasta por $85 \%$ por linfocitos, lo que generaría los niveles observados (Malek, 2003; Tizard, 2013; Kleiveland, 2015). Los niveles obtenidos son comparables a los expresados utilizando antígenos clostridiales y de pasteurelas frente a la evaluación de la expresión in vitro (More et al., 2013; Watanabe et al., 2014; Maximiliano et al., 2018). 
Con relación a la respuesta $\mathrm{Th} 2$, la expresión de la interleucina 4 (IL-4) a las $48 \mathrm{~h}$ de la exposición, solo los grupos Vector (5.62 $\pm 1.12)$ y la combinación Vector $+\mathrm{rF} 17$ (4.67 \pm 0.42 ), mostraron niveles significativamente mayores con relación al control $(2.82 \pm 0.54)$, mientras que a las $72 \mathrm{~h}$ solo la combinación Vector $+\mathrm{rF} 17(5.12 \pm 0.93)$ expresó niveles significativamente mayores. Estos niveles fueron similares a los obtenidos por Maximiliano et al. (2018), quienes entre las 24 y $48 \mathrm{~h}$ obtienen niveles de hasta ocho veces la expresión. No obstante, la proteína rF17 no mostró niveles significativamente mayores, resultado similar al obtenido con el estímulo de antígenos clostridiales (More et al., 2013). IL-4 tiene como función la polarización de linfocitos T vírgenes hacia Th2, lo cual estimula su expansión y activación, siendo producida por Linfocitos T, principalmente (Tizard, 2013). Liu et al. (2009), utilizando un sistema in vivo, obtuvieron niveles de IL-4 predominantes sobre IFN- $\gamma$, expresados en bazo y placas de Peyer al inmunizar ratones BALB/c, utilizando Lactobacillus casei expresando F41 de E. coli enterotoxigénica (ETEC).

La expresión de la interleucina 10 (IL10), en PBMC de alpaca, a las $48 \mathrm{~h}$ de la exposición evidenció que los grupos Vector $(2.88 \pm 1.23)$ y la combinación Vector $+\mathrm{rF} 17$ $(3.67 \pm 0.97)$ mostraron niveles significativamente mayores con relación al control $(0.98 \pm 0.28)$, mientras que a las $72 \mathrm{~h}$ no hubo expresiones significativas. Esta expresión podría explicarse debido a que esta citoquina tiene como función principal la inmunoregulación, y permite la inhibición de macrófagos y células asesinas naturales o $\mathrm{NK}$, disminuyendo la respuesta Th1, actuando frente a niveles altos de IFN- $\gamma$ (Schandené et al., 1994; Tizard, 2013). Se ha demostrado tendencias similares utilizando antígenos clostridiales (More et al., 2013; Tambillo et al, 2014), así como con P6-like de Pasteurella (Maximiliano et al., 2018).

\section{Conclusiones}

El Vector con la proteína recombinante rF17 estimulan la producción de citoquinas de Th1 y Th2, principalmente INT- $\gamma$ y IL-4 en células mononucleares de sangre periférica (PBMC) de alpaca.

\section{Agradecimientos}

Los autores agradecen el financiamiento del Fondo Nacional de Desarrollo Científico, Tecnológico y de Innovación Tecnológica (FONDECYT) por el Programa Doctoral en Medicina Veterinaria (Convenio de Subvención $\mathrm{N},{ }^{\circ}$ 215-2014-FONDECYT).

\section{Literatura Citada}

1. Bihannic M, Ghanbarpour R, Auvray F, Cavalié L, Châtre P, Boury M, Brugère $\mathrm{H}$, et al. 2014. Identification and detection of three new F17 fimbrial variants in Escherichia coli strains isolated from cattle. Vet Res 45: 1-12. doi: 10.1186/s13567-014-0076-9

2. Cid D, Martin-Espada C, Maturrano L, Garcia A, Luna L, Rosadio $R$. 2012. Diarrheagenic Escherichia coli strains isolated from young Peruvian alpacas (Vicugna pacos) with diarrea. In: Perez-Cabal MA, et al. (eds). Production in South American camelids and other fibre animals. p 223-228.

3. Cox E, Melkebeek V, Devriendt B, Goddeeris BM. 2014. Vaccines against enteric coli infections in animals. Caister Academic Press. 304 p.

4. Curtis B, Grassel C, Laufer RS, Sears KT, Pasetti MF, Barry EM, Simon R. 2016. Simple method for purification of enterotoxigenic Escherichia coli fimbriae. Protein Expr Purif 119: 130135. doi: 10.1016/j.pep.2015.11.007 
5. Francis DH, Willgohs JA. 1991. Evaluation of a live avirulent Escherichia coli vaccine for $\mathrm{K} 88+$, $\mathrm{LT}+$ enterotoxigenic colibacillosis in weaned pigs. Am J BVet Res 52: 1051-1055.

6. Friberg D, Bryant J, Shannon W, Whiteside TL. 1994. In vitro cytokine production by normal human peripheral blood mononuclear cells as a measure of immunocompetence or the state of activation. Clin Diagn Lab Immunol 1: 261-268.

7. Gomes-Solecki MJC, Brisson DR, Dattwyler RJ. 2006. Oral vaccine that breaks the transmission cycle of the Lyme disease spirochete can be delivered via bait. Vaccine 24: 44404449. doi: 10.1016/j.vaccine.2005.08.089

8. Heney D, Whicher JT, 1995. Factors affecting the measurement of cytokines in biological fluids: Implications for their clinical measurement. Ann Clin Biochem 32: 358-368. doi: 10.1177/000456329503200402

9. Hur J, Stein BD, Lee JH. 2012. A vaccine candidate for post-weaning diarrhea in swine constructed with a live attenuated Salmonella delivering Escherichia coli K88ab, K88ac, FedA, and FedF fimbrial antigens and its immune responses in a murine model. Can J Vet Res 76: 186-194.

10. Kleiveland CR. 2015. Peripheral blood mononuclear cells. In: The impact of food bioactives on health. Springer. p 161-167.

11. Liu JK, Hou XL, Wei CH, Yu LY, He XJ, Wang GH, Lee JS, Kim CJ. 2009. Induction of immune responses in mice after oral immunization with recombinant Lactobacillus casei strains expressing enterotoxigenic Escherichia coli F41 fimbrial protein. Appl Environ Microbiol 75 :4491-4497. doi: 10.1128/AEM.02672-08

12. Luna-Pineda VM, Reyes-Grajeda JP, Cruz-Córdova A, Saldaña-Ahuactzi $Z$, Ochoa SA, Maldonado-Bernal C, Cázares-Domínguez, V, et al. 2016. Dimeric and trimeric fusion proteins generated with fimbrial adhesins of uropathogenic Escherichia coli. Front
Cell Infect Microbiol 6: 135. doi: 10.3389/ fcimb.2016.00135

13. Luna L, Rivera H, Zanabria V, Rosadio R. 2012. Genotipificación, evaluación toxigénica in vitro y sensibilidad a antibióticos de cepas de Escherichia coli aisladas de casos diarreicos y fatales en alpacas neonatas. Rev Inv Vet Perú 23: 280-288. doi: 10.15381/rivep.v23i3.910

14. Malek TR. 2003. The main function of IL-2 is to promote the development of T regulatory cells. J Leukoc Biol 74: 961965. doi: $10.1189 / \mathrm{jlb} .0603272$

15. Maximiliano J, Maturrano HL, Luna EL, Hurtado CR, Chero OA, Rosadio $\boldsymbol{A R}$. 2018. Evaluación de la inmunogenicidad de una proteína recombinante de una Pasteurella multocida aislada de alpacas con neumonía. Rev Inv Vet Perú 29: 339-348. doi: 10.15381/ rivep.v29i1.14197

16. Mohawk KL, Melton-Celsa AR, Zangari T, Carroll EE, O'Brien AD. 2010. Pathogenesis of Escherichia coli O157:H7 strain 86-24 following oral infection of BALB/c mice with an intact commensal flora. Microb Pathog 48: 131142. doi: 10.1016/j.micpath.2010.01.003

17. Morales S, Paredes D, Pezo D. 2007. Asociación de Rotavirus y Escherichia coli fimbriada como agentes causales de infecciones entéricas en alpacas y enteropatogénica. Rev Inv Vet Perú 18: 150-153. doi: 10.15381/rivep.v18i2.1486

18. More J, Manchego A, Rivera $H$, Sandoval N, Ramírez M, Kim Lam C, Lazaro R, Bardález C. 2013. Expression of cytokines that regulate immune responses in alpaca (Vicugna pacos) blood leukocytes stimulated by clostridial antigens and retinoic acid. Front Immunol 4. doi: 10.3389/ conf.fimmu.2013.02.00318

19. Mori L, Perales R, Rodríguez J, Shiva C, Koga Y, Choquehuanca G, Palacios C. 2014. Molecular identification of Shiga-toxin producing and enteropathogenic Escherichia coli (STEC and EPEC) in diarrheic and healthy young 
alpacas. Adv Microbiol 04: 360-364. doi: 10.4236/aim.2014.47043

20. Nagy B, Fekete PZ. 1999. Enterotoxigenic Escherichia coli (ETEC) in farm animals. Vet Res 30: 259-284. doi: 10.1016/S0928-4249(99)80020-0

21. Odbileg R, Konnai S, Usui T, Ohashi K, Onuma M. 2005. Quantification of llama inflammatory cytokine mRNAs by real-time RT-PCR. J Vet Med Sci 67: 195-198. doi: 10.1292/jvms.67.195

22. Rosadio A, Maturrano H, Pérez J, Luna E. 2012. El complejo entérico neonatal en alpacas andinas. Rev Inv Vet Perú 23: 261-271. doi: 10.15381/rivep.v23i3.908

23. Rosano GL, Ceccarelli EA. 2014. Recombinant protein expression in Escherichia coli: advances and challenges. Front Microbiol 5: 172. doi: 10.3389/fmicb.2014.00172

24. Schandené L, Alonso-Vega C, Willems F, Gérard C, Delvaux A, Velu T, Devos R, de Boer M, Goldman M. 1994. B7/ CD28-dependent IL-5 production by human resting T cells is inhibited by IL10. J Immunol 152: 4368-4374. doi: 10.4049/jimmunol.180.9.5771

25. Sulabh S, Panigrahi M, Kumar S, Varshney R, Verma A, Baba NA, Gupta JP, et al. 2019. Differential cytokine response of Escherichia coli lipopolysaccharide stimulated peripheral blood mononuclear cells in crossbred cattle, Tharparkar cattle and Murrah buffalo - An in vitro study. Spa J Agric Res 17: e0501. doi: 10.5424/sjar/2019171-12599
26. Tambillo GL, Manchego SA, Chiok CKL, Sandoval CN, More BJ, Rivera GH. 2014. Evaluación in vitro de la respuesta leucocitaria de alpacas (Vicugna pacos) en presencia de antígenos clostridiales. Rev Inv Vet Perú 24: 510523. doi: 10.15381/rivep.v24i4.2742

27. Tiels P, Verdonck F, Coddens A, Goddeeris B, Cox E. 2008. The excretion of $\mathrm{F} 18+E$. coli is reduced after oral immunisation of pigs with a FedF and F4 fimbriae conjugate 21542163. Vaccine 26; 2154-2163. doi: 10.1016/j.vaccine.2008.01.054

28. Tizard IR. 2013. Veterinary immunology. Elsevier/Saunders. $568 \mathrm{p}$.

29. Watanabe R, Manchego A, Rivera $\mathrm{H}$. 2014. Expresión in vitro de las interleucinas 2 y 10 de linfocitos de alpacas (Vicugna pacos) en presencia de antígenos clostridiales. Rev Inv Vet Perú 25: 419-429. doi: 10.15381/ rivep.v25i3.10121

30. Whitehead CE, Anderson DE. 2006. Neonatal diarrhea in llamas and alpacas. Small Ruminant Res 61: 207-215. doi: 10.1016/j.smallrumres.2005.07.012

31. Whiteside TL. 1994. Cytokines and cytokine measurements in a clinical laboratory. Clin Diagn Lab Immunol 1: 257-260.

32. Zhang H, Xu Y, Zhang Z, You J, Yang Y, Li X. 2018. Protective immunity of a multivalent vaccine candidate against piglet diarrhea caused by enterotoxigenic Escherichia coli (ETEC) in a pig model. Vaccine 36: 723-728. doi: 10.1016/ j.vaccine.2017.12.026 